\title{
Glioblastoma: Patterns of Recurrence and Efficacy of Salvage Treatments
}

\author{
Jiwon Oh, Arjun Sahgal, Paul Sanghera, May N. Tsao, Phil Davey, Kelvin Lam, \\ Sean Symons, Richard Aviv, James R. Perry
}

\begin{abstract}
Background: It is controversial if distant recurrence of glioblastoma is more common after temozolomide (TMZ) concurrent with radiotherapy (RT). Optimal therapy for patients with recurrent disease after RT/TMZ is unclear. Our purpose was to evaluate recurrence patterns in glioblastoma and the effect of treatment at recurrence upon survival. Methods: We performed a retrospective review of 67 patients with newly diagnosed glioblastoma treated with RT/TMZ between 2003-2007. Statistical analyses included Kaplan-Meier method for survival, and multivariate Cox proportional hazards model for the effect of salvage treatment on survival. Results: 58 patients (86.6\%) recurred locally; 9 patients (13.4\%) had a distant non-contiguous focus of new disease. Median survival(MS) was 17 months; median time-to-progression(TTP) 6.8 months. The local and distant groups had comparable prognostic factors. There was no difference in $\operatorname{MS}(\mathrm{p}=0.35)$ or $\operatorname{TTP}(\mathrm{p}=0.95)$ by location of recurrence. At relapse, 26 patients $(38.8 \%)$ received continuous, dose-intense TMZ, 24(35.8\%) other therapy(4.5\% RT; $20.9 \%$ lomustine+/-procarbazine; $4.5 \%$ etoposide; $1.5 \%$ conventional TMZ; 4.5\% TMZ then lomustine), and 17(25.4\%) were untreated. Dose-intense TMZ was associated with prolonged MS compared to all other patients(21.5 months vs. 12.4 months, $\mathrm{p}=0.019, \mathrm{HR}=3.86,95 \% \mathrm{CI}$ : $1.81-8.22)$ and similar to MS with other chemotherapy regimens(18.8 months, $\mathrm{p}=0.40, \mathrm{HR}=1.30,95 \% \mathrm{CI}$ : $0.65-2.61)$. Conclusion: The pattern of recurrence of glioblastoma treated with RT/TMZ was predominantly local. Second-line treatment with continuous dose-intense TMZ may prolong survival in patients with recurrent glioblastoma. Overall survival is similar to other conventional salvage regimens; however TMZ may be better tolerated. This study is limited by its retrospective nature and potential selection bias. Prospective controlled studies are needed.
\end{abstract}

RÉSUMÉ: Modalités de récidive du glioblastome et efficacité des traitements de rattrapage. Contexte : La possibilité d'une fréquence accrue de récidive à distance du glioblastome après l'administration concomitante de témolomide (TMZ) et de radiothérapie (RT) demeure un sujet de controverse. De même, le traitement optimal des patients qui présentent une récidive après la RT/TMZ n'est pas bien établi. Le but de cette étude était d'évaluer les modes de récurrence du glioblastome et l'effet du traitement de la récidive sur la survie. Méthode : Nous avons effectué une revue rétrospective des dossiers de 67 patients chez qui un diagnostic de glioblastome venait d'être posé et qui ont été traités par la RT/TMZ entre 2003 et 2007 . Nous avons utilisé la méthode de Kaplan-Meier pour analyser les données de survie et le modèle de régression multivariée des risques proportionnels de Cox pour évaluer l'effet du traitement de rattrapage sur la survie. Résultats : Chez 58 patients $(86,6 \%)$ la récidive était locale; 9 patients (13,4\%) ont présenté un nouveau foyer non-contigu à distance. La survie médiane (SM) était de 17 mois et l'intervalle médian entre le traitement initial et la récidive (IR) était de 6,8 mois. Les deux groupes de patients présentaient des facteurs de pronostic comparables. Il n'y avait pas de différence dans la SM ( $p=0,35)$ et l'IR $(\mathrm{p}=0,95)$ selon l'endroit de la récidive. Au moment de la récidive, 26 patients $(38,8 \%)$ recevaient du TMZ de façon continue à dose élevée, 24 $(35,8 \%)$ recevaient un autre traitement (RT chez 4,5\% ; lomustine \pm procubaine chez 20,9\% ; étoposide chez 4,5\% ; TMZ conventionnel chez 1,5\% ; TMZ suivi de lomustine chez $4,5 \%)$ et 17 patients $(25,4 \%)$ ne recevaient aucun traitement. Le traitement intensif par le TMZ était associé à une SM prolongée par rapport à celle des autres patients $(21,5$ mois versus 12,4 mois $; \mathrm{p}=0.019 ;$ rapport de risques $=3,86 ;$ IC à $95 \%: 1,81$ à 8,22$)$ et similaire à la SM des autres régimes posologiques de chimiothérapie $(18,8$ mois $; \mathrm{p}=0,40 ;$ rapport de risques $=1,30 ; \mathrm{IC}$ à $95 \%: 0,65$ à 2,61$)$. Conclusion : Le mode de récidive du glioblastome traité par la RT/TMZ était surtout local. Le traitement de seconde intention par le TMZ à dose élevée continue pourrait prolonger la survie chez les patients qui présentent une récidive. La survie globale est similaire à celle d'autres régimes posologiques de rattrapage conventionnels. Cependant, il est possible que le TMZ soit mieux toléré. Le fait que cette étude soit rétrospective et qu'il existe potentiellement des biais de sélection en limitent la portée. Seules des études prospectives contrôlées pourront élucider la question.

Can. J. Neurol. Sci. 2011; 38: 621-625

Glioblastoma is the most common malignant primary brain tumour in adults. Prior to the use of temozolomide (TMZ), there were limited chemotherapeutic options for patients with glioblastoma and the standard of care consisted of maximal surgical resection followed by radiation therapy (RT). Since the publication of the randomized controlled trial of radiation alone versus radiation with concurrent and adjuvant TMZ (Stupp regimen), TMZ is now widely accepted as the standard of care in the upfront therapy of patients with glioblastoma ${ }^{1}$.

Despite the unequivocal benefit of TMZ chemotherapy in newly-diagnosed glioblastoma, most tumours recur.
Before the use of TMZ, with radiation alone, the radiographic pattern of recurrence of glioblastoma was shown to be

From the Divison of Neurology (JO, KL, JRP), Departments of Medicine, and Radiation Oncology (AS, PS, MNT, PD), Division of Neuroradiology (SS, RA), Department of Medical Imaging, Odette Cancer Centre, Sunnybrook Health Sciences Centre, Toronto, Ontario, Canada.

Received December 20, 2010. Final Revisions Submitted February 2, 2011 Correspondence to: Jiwon Oh, c/o Office of Dr. James R Perry, Sunnybrook Health Sciences Centre, University of Toronto, A402, 2075 Bayview Avenue, Toronto, Ontario, M4N 3M5, Canada. 
predominately "local", or in close proximity to the site of the original cancer burden with a number of studies reporting 80$90 \%$ local recurrence. Using whole brain radiotherapy alone, Hochberg et al reported $80 \%$ local recurrence, while for involved-field radiotherapy, Lee et al reported $89 \%$ local recurrence ${ }^{2,3}$. Currently, there is limited literature regarding recurrence patterns of glioblastoma in the era of contemporary treatment with RT/TMZ.

Furthermore, the optimal management of patients with tumour recurrence after upfront treatment with TMZ remains unclear. There is emerging evidence that re-treatment with continuous dose-intense TMZ at $50 \mathrm{mg} / \mathrm{m}^{2} /$ day may be a welltolerated, valuable therapeutic option in recurrent glioblastoma patients, but this has not been compared retrospectively or otherwise to standard chemotherapy regimens ${ }^{4}$.

The purpose of this study was to assess for a qualitative change in the radiographic patterns of recurrence in patients with glioblastoma treated with radiation therapy (RT) and concurrent and adjuvant TMZ chemotherapy, and to report the effects of treatment choice at the time of recurrence upon survival.

\section{METHODS}

\section{Patient Eligibility}

This project was approved by the Research Ethics Board at the Sunnybrook Health Sciences Centre, and informed consent was obtained from all study subjects and/or guardians at the time of registry into a prospective database. All patients with newly diagnosed glioblastoma receiving RT/TMZ between July 2003December 2007 at the Sunnybrook Health Sciences Centre, Toronto, Ontario, Canada were identified. Temozolomide was administered at a dose of $75 \mathrm{mg}$ per $\mathrm{m}^{2}$ concurrent with daily radiotherapy and followed by $150 \mathrm{mg}-200 \mathrm{mg}$ per $\mathrm{m}^{2}$ for five days every 28 days. The duration of adjuvant cycles of TMZ varied, and was individualized to each patient depending on his/her tumour response and clinical status. Inclusion criteria consisted of: Patients $>18$ years or age, pathologically confirmed glioblastoma, treatment with RT/TMZ, and unequivocal tumour recurrence meeting RECIST criteria ${ }^{5}$. Patients with tumour recurrence not meeting RECIST criteria were included if there was associated clinical deterioration. Patients with Grade III anaplastic astrocytoma were included only if the initial radiological features showed large areas of necrosis suggestive of glioblastoma, since the prognosis in such patients has been reported to be similar to glioblastoma ${ }^{6}$. Patients lacking adequate contrast-enhanced baseline and recurrence scans for analysis were excluded, as were those treated with experimental agents and protocols. Finally, patients with early progression (within eight weeks of completing concurrent chemoradiation) were followed to ensure that there was further radiological or clinical deterioration within the first six months. Early recurrent lesions with subsequent resolution within six months ("pseudoprogressive" lesions) were not included in the analysis ${ }^{7}$. Overall, a total of 67 patients were included in the analysis.

A retrospective chart review was performed to collect data which included: age, performance status, extent of surgery, pathology, radiation dose, number of adjuvant TMZ cycles, use of salvage therapy, date of tumour recurrence, and date of death. computed tomogram (CT) or magnetic resonance imaging
(MRI) scans at baseline and first recurrence were reviewed by a neurologist, neuro-radiologist, and neuro-radiation oncologist on all patients.

\section{Analysis of Recurrence Patterns}

In each patient, a baseline post-operative scan prior to the initiation of radiotherapy was compared to the scan of the tumour at first recurrence. The site of the tumour at first recurrence was then compared to the original site of the tumour. We defined each separate focus of recurrent tumour as "local" if the $>80 \%$ contrast enhancing lesion arose within $2 \mathrm{~cm}$ of the border of the original tumour; (Figures 1a,b) "marginal" if $>80 \%$ of the recurrent contrast-enhancing lesion arose between 2 and $2.5 \mathrm{~cm}$ of the original tumour, and "distant" if $>80 \%$ of the recurrent contrast-enhancing lesions arose $>2.5 \mathrm{~cm}$ from the site of the original tumour (Figures 1c, d). The demarcation of 2-2.5 $\mathrm{cm}$ as a marginal failure is based on standard margins applied to the contrast enhancing mass to be encompassed in the high dose radiation volume as per the Stupp regimen. In tumours with multicentric (multifocal) recurrences, if any of the foci were greater than $2.5 \mathrm{~cm}$ from the original tumour, these tumours were also defined as "distant".

\section{Statistical Analysis}

Statistical analyses included Kaplan-Meier method for survival and time-to progression. The one-sided log-rank test

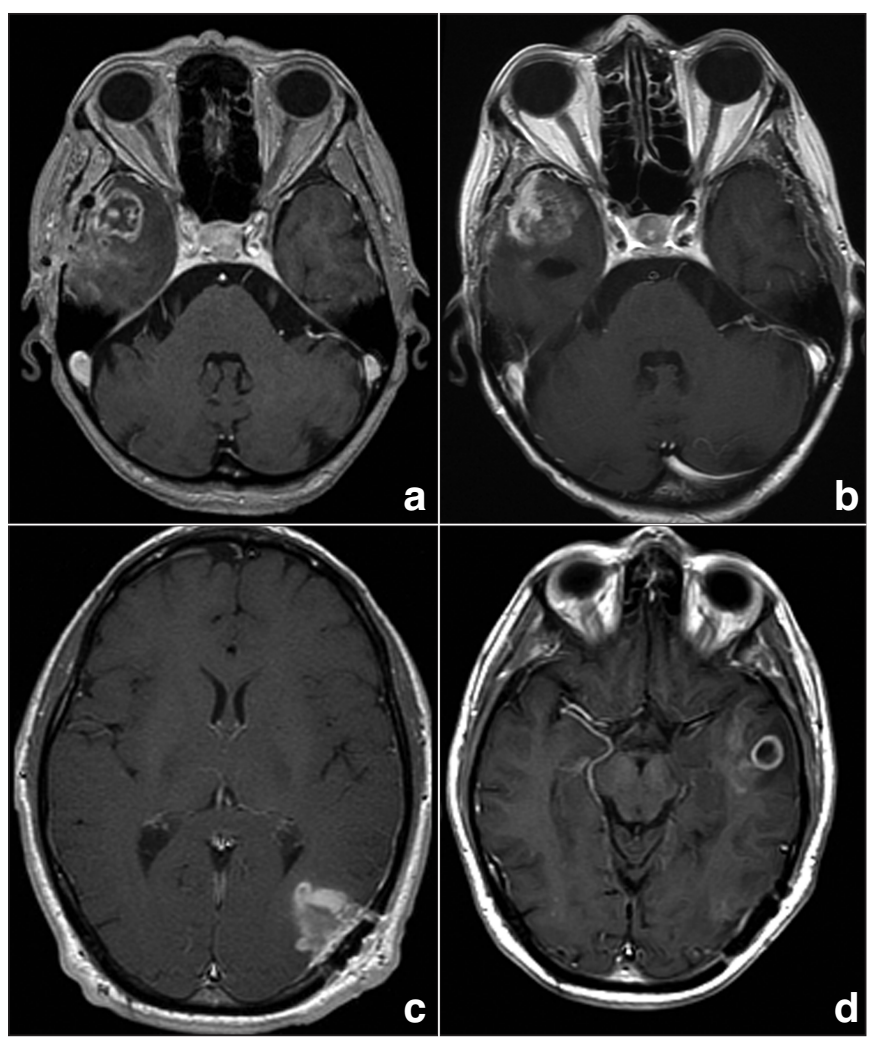

Figure 1: a) "Local" recurrence (at baseline); b) "Local" recurrence (at recurrence); c) "Distant" recurrence (at baseline); d) "Distant" recurrence (at recurrence). 


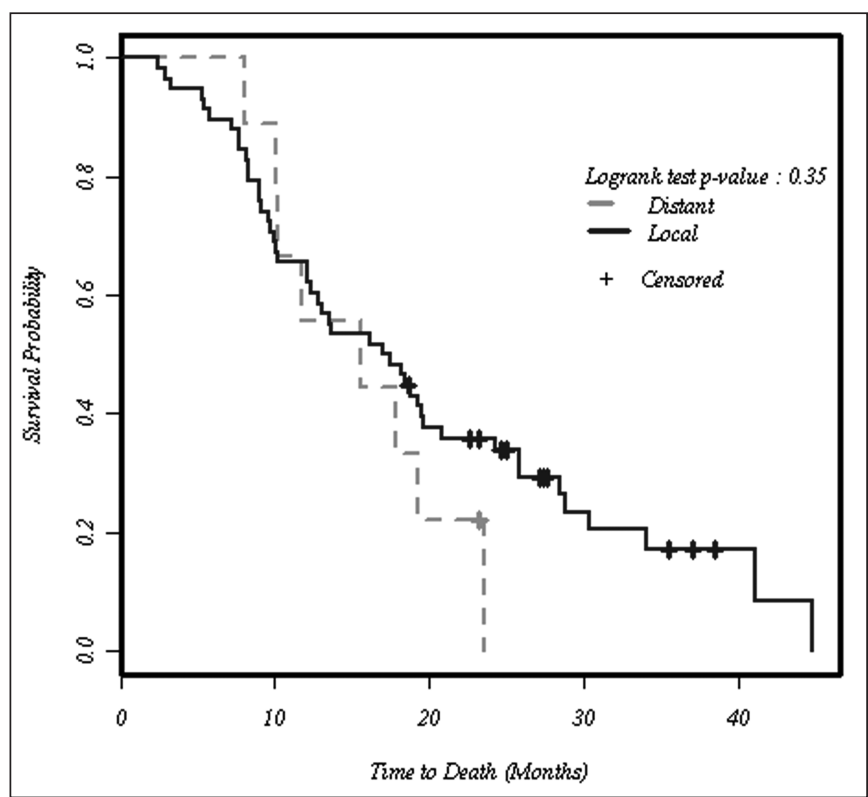

Figure 2: Overall survival by pattern of recurrence.

was used to assess for differences in the prognostic factors of age, sex, and extent of surgery. The Cox proportional hazards model was used to describe the effect of recurrence pattern ("local" vs "distant") on survival and time-to-progression (TTP), and to describe the effect of salvage treatment on overall survival. Other variables included in both Cox proportional hazards models were: age, sex, degree of surgical resection, and

Table 1: Patient characteristics

\begin{tabular}{l|l|l}
\hline Characteristic & N & \% \\
\hline Pattern of Recurrence & & \\
\hline Local & 58 & 86.6 \\
\hline Distant & 9 & 13.4 \\
\hline Age & & \\
\hline$>=60$ & 22 & 32.8 \\
\hline$<60$ & 45 & 67.2 \\
\hline Sex & & \\
\hline M & 40 & 59.7 \\
\hline F & 27 & 40.3 \\
\hline $0-2$ & & \\
\hline $3-4$ & 35 & 52.2 \\
\hline Degree of surgical resection & 32 & 47.8 \\
\hline Biopsy & & \\
\hline Debulking & 14 & 20.9 \\
\hline Resection & 44 & 65.7 \\
\hline
\end{tabular}

ECOG=Eastern Cooperative Oncology Group

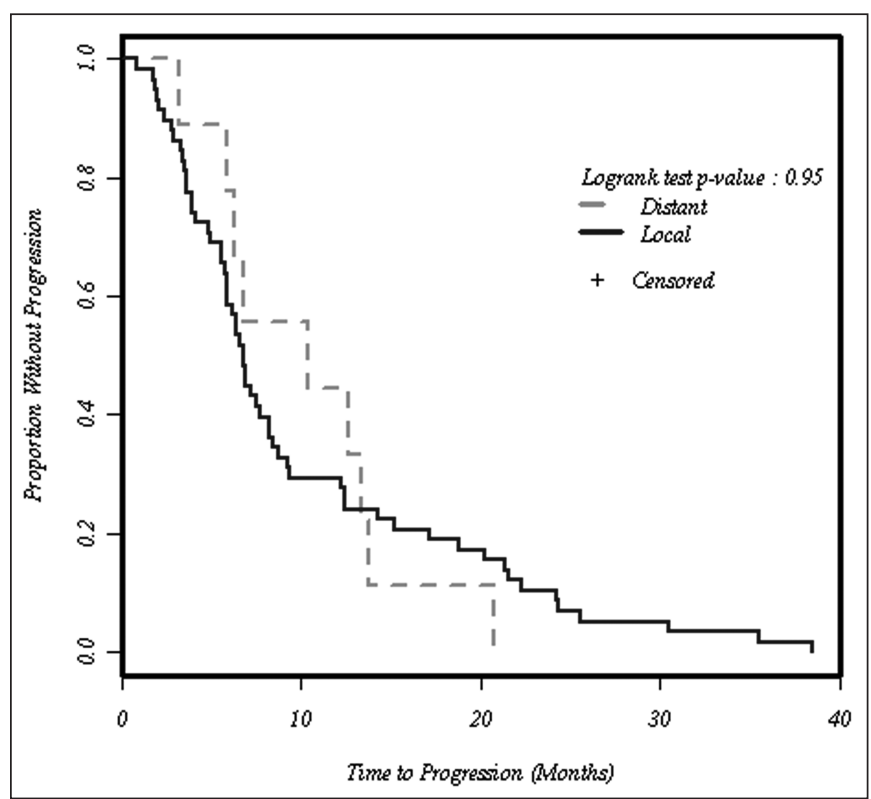

Figure 3: Time to progression by pattern of recurrence

performance status. Alpha was set at 0.05 . Tests were considered statistically significant if p-values were less than 0.025 .

\section{RESULTS}

Of 111 total patients identified, 67 patients met inclusion criteria, and 44 patients were excluded from the analysis. Reasons for exclusion included: no recurrence (6 patients), inadequate post-op scan (17 patients), clinical deterioration at outside hospital with no scan available for analysis (21 patients). The baseline characteristics of the 67 patients included in the study are outlined in Table 1 .

Of 67 total patients analyzed, the pattern of recurrence was determined to be "local" in 58 patients $(86.6 \%)$, while 9 patients $(13.4 \%)$ had recurrence which included a distant non-contiguous focus of disease. There were no significant differences in the prognostic factors of age, performance status, and degree of surgical resection when comparing the groups with "local" versus "distant" recurrence (Table 2). Median survival in all patients was 17.0 months (95\% CI 12.1 - 19.5 months), and median time to progression was 6.8 months (95\% CI 5.9-8.5 months). When the "local" and "distant" recurrence groups were compared with respect to the factors of age, performance status, and degree of resection, the groups were comparable with no significant difference in any of these variables (Table 2). When the "local" and "distant" groups were compared with respect to median survival and TTP, there was no evident difference in these variables when stratified by recurrence pattern (Figures 2 and $3, p=0.35$ for overall survival, $\mathrm{p}=0.95$ for TTP).

At recurrence, 26 patients $(38.8 \%)$ received continuous, doseintense TMZ, 24 patients $(35.8 \%)$ other salvage regimens, and 17 patients $(25.4 \%)$ were untreated. Specific salvage regimens used are outlined in Table 3. The median survival of patients treated with continuous dose-intense TMZ was prolonged compared to all other patients (21.5 months vs. 12.4 months, 
Table 2: Prognostic factors in the "local" vs. "distant" groups are comparable

\begin{tabular}{l|l|l}
\hline & Local & Distant \\
\hline Median age (years) & 56 & 52 \\
\hline Performance Status & & \\
\hline ECOG 0-2 & 30 & 5 \\
\hline ECOG 3-4 & 28 & 4 \\
\hline Degree of resection & & \\
\hline Biopsy & 14 & 0 \\
\hline Decompression & 36 & 8 \\
\hline Resection & 8 & 1 \\
\hline
\end{tabular}

ECOG=Eastern Cooperative Oncology Group

$\mathrm{p}=0.019, \mathrm{HR}=3.86,95 \% \mathrm{CI}: 1.81-8.22)$. Median survival was similar to those treated with other salvage chemotherapy regimens (18.8 months, $\mathrm{p}=0.40, \mathrm{HR}=1.30,95 \% \mathrm{CI}: 0.65-2.61)$, (Figure 4).

\section{Discussion}

Since the adoption of TMZ chemotherapy in the treatment of glioblastoma, the overall survival of patients has improved ${ }^{1}$. Despite encouraging developments, most tumours ultimately recur. We hypothesized that the improved outcomes with RT/TMZ would yield a shift in patterns of recurrence given the mechanism of action of TMZ.

Temozolomide is known to chemically methylate DNA, forming O6-guanine adducts. These compounds are mainly

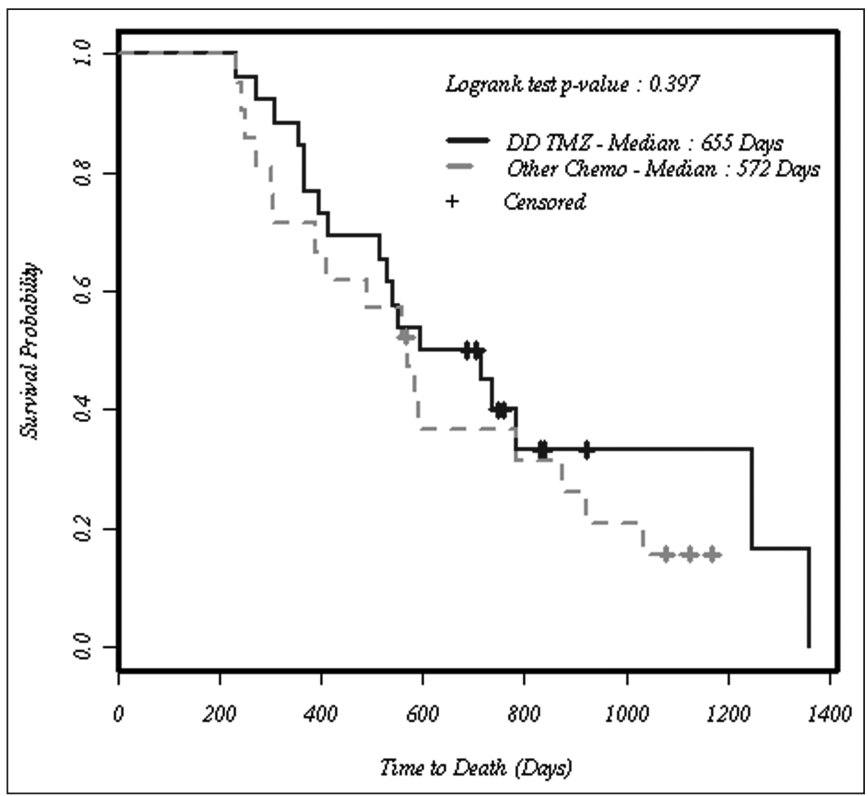

Figure 4: : Overall survival of patients receiving dose-intense TMZ vs. patients receiving other salvage chemotherapy regimens.
Table 3: Salvage regimens after tumour recurrence

\begin{tabular}{l|l|l}
\hline Salvage Regimen & N (\%) & Notes \\
\hline Chemotherapy & $47(70.1)$ & \\
\hline Dose-intense TMZ & $26(38.8)$ & $\begin{array}{l}1 \text { patient also received } \\
\text { salvage surgery }\end{array}$ \\
\hline CCNU & $11(16.4)$ & $\begin{array}{l}\text { 2 patients also received } \\
\text { salvage surgery }\end{array}$ \\
\hline $\begin{array}{l}\text { CCNU + } \\
\text { procarbazine }\end{array}$ & $3(4.5)$ & \\
\hline Etoposide & $3(4.5)$ & $\begin{array}{l}1 \text { patient also received } \\
\text { RT/surgery }\end{array}$ \\
\hline Conventional TMZ & $1(1.5)$ & \\
\hline $\begin{array}{l}\text { Conventional TMZ } \\
\text { then etoposide }\end{array}$ & $3(4.5)$ & \\
\hline RT alone & $3(4.5)$ & \\
\hline No Treatment & $17(25.4)$ & \\
\hline
\end{tabular}

TMZ=temozolomide, $\mathrm{CCNU}=$ lomustine, $\mathrm{RT}$ =radiation therapy

responsible for TMZ's toxic effects on malignant cells by overcoming the cell's DNA repair mechanisms, which results in cell death ${ }^{8}$. Temozolomide may have additional biological effects to account for its clinical efficacy when used in combination with radiation therapy. There is some evidence that TMZ may have radio-sensitizing effects, and that TMZ can act synergistically with radiation to enhance radiation-mediated double-stranded DNA breaks and to suppress DNA repair pathways after radiation exposure ${ }^{9}$. In addition, the potential for TMZ to reduce tumour cell mobility has been described in the preclinical literature ${ }^{10}$. If $\mathrm{TMZ}$ does have such additional biological effects, one could speculate that better local tumour control would be achieved with the use of TMZ in combination with RT, which would result in an increase in distant recurrences beyond the high dose radiation volume.

Currently, there is limited literature regarding recurrence patterns of glioblastoma in the era of contemporary treatment with RT/TMZ, with three recent articles report conflicting results ${ }^{10-12}$. Using a subset of 30 patients from the pivotal EORTC/NCIC-CTG trial and MRI software, Wick et al demonstrated that there was no difference in the proportion of patients with distant recurrence $(20 \%)$ between the RT/TMZ treatment group and the control group ${ }^{11}$. In contrast, Brandes et al analyzed a group of 95 patients who had received concurrent and adjuvant TMZ with radiation therapy and found only $72.2 \%$ local recurrence, with $21.5 \%$ distant recurrence. They suggested an increase in distant recurrence patterns in comparison to historical controls prior to the use of TMZ. Interestingly, Brandes et al found that MGMT status correlated with tumour recurrence pattern, because patients with methylated MGMT promoters were more likely to have distant recurrence ${ }^{12}$.

Recently, Milano et al reported a predominately local pattern of recurrence in 54 patients treated with RT/TMZ. In their cohort, they showed an increasing proportion of distant recurrences with prolonged survival, and that central recurrence 
developed earlier than distant recurrence. The authors postulated that these observations may reflect the greater susceptibility of patients to develop distant recurrences as a result of the prolonged survival offered by TMZ's radio-sensitizing effects ${ }^{13}$.

Using similar methodology to define recurrence patterns as these recent reports, we found that the pattern of recurrence of glioblastoma in patients treated with TMZ/RT chemoradiation was predominately local $(86.6 \%)$, similar to what was reported in the historical literature. Furthermore, in contrast to the recent findings reported by Milano et $\mathrm{al}^{13}$, we found that there was no difference in the median TTP in the "local" and "distant" recurrence groups.

At tumour recurrence, the majority of patients in our study were treated with some sort of salvage treatment such as chemotherapy, radiation, or surgery. Currently, there is no consensus on the optimal medical management of patients who recur after upfront TMZ. There is emerging literature on the utility of daily TMZ (50 mg/m²/day orally, continuously) as a potential treatment option in these patients. The altered dosing regimen of $\mathrm{TMZ}$ is postulated to further suppress MGMT activity, and may have anti-angiogenic effects to account for its clinical efficacy ${ }^{14,15}$. A recent Phase II trial by Perry et al showed that continuous dose-intense TMZ was a well-tolerated, valuable salvage option in glioblastoma, and up to $36 \%$ of patients achieved a six-month progression-free survival ${ }^{16}$.

When we compared our cohort of patients treated with continuous dose-intense TMZ, the median survival of patients was comparable to other established salvage chemotherapy regimens. This suggests that DD-TMZ may not be inferior to conventional salvage chemotherapies. A randomized study is required to determine efficacy as compared to standard salvage regimens. However, continuous, dose-intense TMZ has the added benefit of ease of administration ${ }^{4}$.

This study has a number of other limitations. First, the data were collected retrospectively, and the power of the study was limited due to the small size of the patient population analyzed. In addition, we were unable to use uniform imaging modalities (CT vs MRI) in the imaging analysis, as the scans were collected retrospectively. However, this is unlikely to have affected the designation of recurrent tumours as "local" versus "distant" as these designations were made based on distance from the site of the original tumour, which is easily measurable using anatomical landmarks, regardless of which imaging modality was used (CT or MRI). Finally, our findings were limited by the fact that there was no internal "control" group to which we could compare our patient population's recurrence patterns. Therefore, we compared our results to the historical literature on recurrence patterns. Finally, our ability to draw definitive conclusions about the efficacy of continuous dose-intense TMZ is limited by the retrospective nature of this study and the potential for treatment selection bias, especially those patients selected for daily TMZ versus other therapies.

Our findings contribute to the limited literature on patterns of recurrence and treatment of recurrent glioblastoma in the TMZ era of chemoradiation. Large, prospective trials are needed to definitively characterize patterns of recurrence in glioblastoma patients treated with RT/TMZ. Randomized comparative trials would be useful to ascertain the optimal medical management of recurrent glioblastoma after upfront exposure to TMZ.

\section{ACKNOWLEDGEMENTS}

Brain Tumour Foundation of Canada Crolla Chair of Brain Tumour Research

*Note: Figures 2-4 created using Program “ $R$ ”: R Development Core Team (2010). R: A language and environment for statistical computing. $R$ Foundation for Statistical Computing, Vienna, Austria. ISBN 3-900051-07-0, URL: http://www.R-project.org.

\section{REFERENCES}

1. Stupp R, Mason WP, Van den Bent MJ, et al. Radiotherapy plus concomitant and adjuvant temozolomide for glioblastoma. $\mathrm{N}$ Engl J Med. 2005;352(10):987-96.

2. Hochberg FH, Pruitt A. Assumptions in the radiotherapy of glioblastoma. Neurology. 1980;30(9):907-11.

3. Lee SW, Fraass BA, Marsh LH, et al. Patterns of failure following high-dose 3-D conformal radiotherapy for high-grade astrocytomas: a quantitative dosimetric study. Int J Radiat Oncol Biol Phys. 1999;43(1):79-88.

4. Perry JR, Rizek P, Morrison T, Morrison M, Cashman R. Temozolomide rechallenge in recurrent malignant glioma using a continuous temozolomide schedule: the "rescue" approach. Cancer. 2008;113(8):2152-7.

5. Therasse P, Arbuck SG, Eisenhauer EA, et al. New guidelines to evaluate the response to treatment in solid tumors. European Organization for Research and Treatment of Cancer, National Cancer Institute of the United States, National Cancer Institute of Canada. J Natl Cancer Inst. 2000;92(3):205-16.

6. Burnet NG, Lynch AG, Jefferies SJ, et al. High grade glioma: imaging combined with pathological grade defines management and predicts prognosis. Radiother Oncol. 2007;85:371-8.

7. Sanghera P, Perry J, Sahgal A, et al. Pseudoprogression following chemoradiotherapy for glioblastoma multiforme. Can J Neurol Sci. 2010;37(1):36-42.

8. van Nifterik KA, van den Berg J, Stalpers LJ, et al. Differential radiosensitizing potential of temozolomide in MGMT promoter methylated glioblastoma multiforme cell lines. Int J Rad Onc Biol Phys. 2007;69(4):1246-53.

9. Chakravarti A, Erkkinen MG, Nestler U, et al. Temozolomidemediated radiation enhancement in glioblastoma: a report on underlying mechanisms. Clin Cancer Res. 2006;12(15):4738-46.

10. Wick W, Wick A, Schulz JB, Dichgans J, Rodemann HP, Weller M. Prevention of irradiation-induced glioma cell invasion by temozolomide involves caspase-3-activity and cleavage of focal adhesion kinase. Cancer Res. 2002;62(6):1915-19.

11. Wick W, Stupp R, Beule A, et al. A novel tool to analyze MRI recurrence patterns. Neuro Oncol. 2008;10(6):1019-24.

12. Brandes AA, Tosoni A, Franceschi E, et al. Recurrence pattern after temozolomide concomitant with and adjuvant to radiotherapy in newly diagnosed patients with glioblastoma: correlation with MGMT promoter methylation status. J Clin Oncol. 2009;27(8): 1275-9.

13. Milano MT, Okunieff P, Donatello RS, et al. Patterns and timing of recurrence after temozolomide-based chemoradiation for glioblastoma. Int J Radiat Oncol Biol Phys. 2010;78(4):1147-55.

14. Tolcher AW, Gerson SL, Denis L, et al. Marked inactivation of O6alkylguanine-DNA alkyltransferase activity with protracted temozolomide schedules. Br J Cancer. 2003;88(7):1004-11.

15. Kerbel RS, Kamen BA. The anti-angiogenic basis of metronomic chemotherapy. Nat Rev Cancer. 2004;4(6):423-6.

16. Perry JR, Bélanger K, Mason WP, et al. Phase II trial of continuous dose-intense temozolomide in recurrent malignant glioma: RESCUE study. J Clin Onc. 2010;28(12):2051-7. 